\section{Bases conceituais para uma estratégia de gestão: o caso da Rede Nacional de Bancos de Leite Humano}

\author{
The management strategy of the Brazilian \\ National Network of Human Milk Banks
}

Paulo Ricardo da Silva Maia 1

Franz Reis Novak 1

João Aprígio Guerra de Almeida

Danielle Aparecida da Silva 1

\footnotetext{
1 Instituto Fernandes

Figueira, Fundação Oswaldo

Cruz, Rio de Janeiro, Brasil.

Correspondência

P. R. S. Maia

Instituto Fernandes Figueira,

Fundação Oswaldo Cruz.

Av. Rui Barbosa 716,

Rio de Janeiro, $R J$

22250-020, Brasil.

pmaia@fiocruz.br
}

\section{Abstract}

The Brazilian National Network of Human Milk Banks (REDEBLH), with its headquarters in the Oswaldo Cruz Foundation in Rio de Janeiro, is experiencing rapid growth. The Network's activity has been acknowledged by the World Health Organization and received the Sasakawa Health Award in 2001 for best public health project. One of the main challenges is to ensure continuing development of competencies to respond to the needs arising from such growth. A new strategy is being pursued to deal with the Network's management issues. This article aims to develop a conceptual framework to contribute to the elaboration of a theoretical framework for new management strategies in the REDEBLH. Using such concepts, the aim is to draw on the typology of networks described in the specialized literature to identify the institutional profile of the REDEBLH. Based on the understanding that it is necessary to identify and understand the processes occurring within networks, and after which to consider management-related issues, the study used a proposal developed for the formation of innovation networks as its analytical tool.

Milk Banks; Health Policy; Management Capacity

\section{Introdução}

A Rede Nacional de Bancos de Leite Humano (REDEBLH) é um programa do Ministério da Saúde (MS) que tem ocupado importante espaço na área da saúde pública do Brasil. Dela fazem parte mais de 160 Bancos de Leite Humano (BLH), distribuídos por todo o país. Hoje existe evidente reconhecimento nacional sobre os avanços na saúde infantil obtidos pela sua implementação. A rede opera através da doação voluntária de leite humano. O leite é destinado para bebês prematuros, de baixo peso, ou hospitalizados em Unidades de Tratamento Intensivo (UTI) Neonatal. No período de 1998 a 2001, foram captados 217 mil litros de leite, o que possibilitou nutrir 288 mil recém-nascidos prematuros. No mesmo período, foram assistidas 1.390.000 mulheres com ações de incentivo ao aleitamento materno (Rede de Bancos de Leite Humano: http:www.redeblh.fiocruz.br, acessado em 29/Abr/2004). Também foram observados benefícios econômicos na medida em que se estima a diminuição na importação de leite artificial, antes necessário para alimentar esses recém-nascidos.

Conforme Almeida 1, o grande desafio, no momento, é dar continuidade ao desenvolvimento de competências para uma nova forma de pensar as questões relacionadas à amamentação. Assim destaca três questões relevantes: a construção de vias que facilitem o acesso dos 
profissionais aos novos saberes; a definição de caminhos que possibilitem o desenvolvimento científico e tecnológico e a substituição do discurso ideológico da amamentação por posições solidamente ancoradas nos diferentes campos do saber.

Por outro lado, a REDEBLH vem experimentando um rápido desenvolvimento que exige respostas importantes do ponto de vista da sua gestão.

A implementação de alternativas que universalizem o acesso ao conhecimento, onde quer que existam BLH em funcionamento, também necessita de novas formas de gestão. Para tanto, pode-se utilizar instrumentos conceituais já disponíveis na área, buscando-se aprofundar os estudos que levem a uma compreensão mais ampliada do funcionamento da REDEBLH.

$O$ rápido avanço tecnológico requer também ágeis mecanismos de gestão para atender a nova dinâmica das transformações políticas, sociais e econômicas. De natureza cada vez mais diversificada, as inovações tecnológicas produzem efeitos modificadores nos sistemas de produção, de serviços e na sociedade de um modo geral. Segundo Peci 2, a utilização de recursos especializados, aliada ao grande volume de processamento de informação, bem como a prevalência de trocas baseadas em personalização são fatores determinantes na proliferação de estruturas organizacionais baseadas no modelo de redes.

O presente estudo tem como objetivos desenvolver um quadro teórico-metodológico que contribua para formulação de políticas na área de gestão da REDEBLH. Busca-se também identificar os principais atores que interagem na REDEBLH por meio da utilização da proposta de Rogers ${ }^{3}$ sobre a difusão e formação de redes de inovação.

\section{Caracterização do objeto de estudo}

Conforme Freitas 4, não se pode pensar em organizações independentes do contexto e da época em que se situam. Isso significa que as organizações devem ser compreendidas dentro de um espaço social e de uma época específica, constituindo-se assim um formato sócio-histórico. Partindo de uma perspectiva histórica, entendida como sucessão de ciclos de vida organizacional do processo de construção da REDEBLH, busca-se delimitar nosso objeto de estudo.
Remontam ao início da década de quarenta os esforços de organização dos BLH no Brasil. Localizado no Instituto Nacional de Puericultura, que mais tarde viria a se transformar no atual Instituto Fernandes Figueira (IFF) da Fundação Oswaldo Cruz (FIOCRUZ), o primeiro BLH implantado no país tinha como objetivos coletar e distribuir leite humano. Eram considerados receptores formais bebês prematuros, crianças com carências nutricionais importantes, ou ainda os que apresentassem intolerância ao leite artificial. Entretanto, segundo Almeida 1, os BLH também surgiram como resposta às falhas do paradigma do desmame comerciogênico, que havia substituído as tradicionais amas-de-leite. Esse pensamento predominou como fator determinante na implantação de novos bancos de leite até metade dos anos 80 e, nesse período, verificou-se modesto aumento numérico de unidades. A partir de 1985 , verifica-se maior rapidez no surgimento de BLH. No intervalo de seis anos (1985 a 1990), foram instalados 47 . Na década seguinte, esse número ultrapassaria as cem unidades.

Há questões, entretanto, que transcendem os aspectos acima assinalados. O surgimento de novo paradigma na concepção e forma de atuação dos BLH, que predomina a partir desse período, é fortemente influenciado pela atuação de profissionais altamente qualificados que neles atuam. O grupo se organizou através da diferenciação e ampliação do espaço de atuação tradicional dos BLH. Avançou na atividade acadêmica por intermédio da formação e permanente qualificação de recursos humanos de nível médio, superior e de pós-graduação stricto sensu. Atividades de investigação científica e de desenvolvimento tecnológico passaram também a fazer parte do arco de sustentação acadêmica para o novo projeto que então se iniciava. Essa ampliação também significaria nova articulação com o campo da saúde coletiva bem como novo espaço de atuação na própria formulação da Política Nacional de Aleitamento Materno. Estava, dessa forma, sedimentado o caminho para sustentação de um novo modelo de gestão e de atuação dos BLH.

Em 1998, a FIOCRUZ, através do BLH do IFF, passou a coordenar a elaboração e implantação do projeto REDEBLH cujo objetivo pode assim ser descrito: nortear a formulação, implementação e acompanhamento da política estatal no âmbito de atuação dos BLH em todo o território brasileiro. Em articulação com o MS, o projeto apontava para ampliação gra- 
dual da rede, tendo como objetivo a atuação interativa e compartilhada de todas as unidades participantes. A partir de então, é possível observar importante crescimento quali-quantitativo dos BLH, associado a uma atuação cada vez mais diferenciada. A rede começou a ser criada com sucesso. Cresceram os investimentos em pesquisa permitindo que o BLH do IFF, agora Centro de Referência Nacional, desenvolvesse métodos de controle de qualidade tipicamente adaptados às necessidades nacionais, que eram seguros e sensíveis o suficiente para serem praticados na rotina. O custo de análise por amostra diminuiu, e as técnicas de processamento foram adaptadas a modelos de alta confiabilidade e também de baixo custo. Essa prática possibilitou enfrentar, com segurança, possíveis agravos e riscos decorrentes do surgimento da AIDS. Por essa razão, enquanto, em várias regiões do mundo, bancos de leite foram fechados por temor às questões de segurança operacional e risco biológico, o Brasil viveu um franco processo de expansão.

A estruturação da REDEBLH complementa-se com as referências regionais ou estaduais formalizadas através de convênios de cooperação firmados entre a FIOCRUZ e as Secretarias Estaduais de Saúde. É dessa forma que se estabelecem as articulações e criam-se os mecanismos de interlocução e cooperação técnico-científica com os BLH participantes da rede.

No momento, a REDEBLH conta com mais de 160 unidades em operação, e sua configuração geográfica pode ser observada na Tabela 1 . Nota-se que aproximadamente $70 \%$ dos BLH estão concentrados nas regiões Sudeste e Sul. Contudo, conhecer apenas o processo de institucionalização da REDEBLH não é suficiente para compreender o funcionamento e seu conseqüente modelo de gestão.

Tabela 1

Distribuição da Rede Nacional de Bancos de Leite Humano por região geográfica, 2003.

\begin{tabular}{lc}
\hline Região & Bancos de leite humano \\
\hline Norte & 6 \\
Nordeste & 34 \\
Sudeste & 80 \\
Sul & 23 \\
Centro-Oeste & 20 \\
Total & 163 \\
\hline
\end{tabular}

\section{Referencial teórico}

De acordo com Castells 5, a economia informacional/global caracteriza-se pela combinação de uma estrutura permanente, uma geometria variável e polarizada ao longo de um eixo que opõe áreas prósperas e ricas, em informação e conhecimento, e áreas empobrecidas, sem valor econômico, e atingidas pela exclusão social. É nesse cenário que ganha importância o novo modelo de estrutura organizacional baseado em redes.

O pressuposto do estudo é o de que existem, pelo menos, duas possibilidades analíticas para compreensão do funcionamento de redes.

Na primeira, entende-se sua dinâmica a partir das características de seu processo produtivo para geração de bens ou serviços. Essa opção implica privilegiar questões relacionadas à estrutura e hierarquia organizacional das redes. O modo de produção e as oportunidades de mercado são elementos centrais. São operados conceitos da teoria das organizações, e sua aplicabilidade tem sido observada em estruturas empresariais voltadas para o lucro financeiro. Observa-se também a configuração estética da rede de forma passiva sem buscar elementos de animação.

A segunda, ao contrário, coloca animação como essência para a compreensão das redes enquanto seu funcionamento. Nesse entendimento, “a questão é sempre a de reatar o nó górdio atravessando, tantas vezes quantas forem necessárias, o corte que separa os conhecimentos exatos e o exercício do poder, digamos a natureza e a cultura ...nosso meio de transporte é a noção de tradução ou de rede. Mais flexível que a noção de sistema, mais histórica que a de estrutura, mais empírica que a de complexidade, a rede é o fio de Ariadne destas histórias confusas" 6 (p. 9).

Esses dois campos teóricos simbolizam, portanto, modos diferentes de compreender conceitualmente a idéia de rede, certamente com implicações diretas no modelo de gestão.

Nesse sentido, é fundamental identificar conceitos que definem configurações possíveis de redes para então estabelecer a identidade funcional da REDEBLH.

Há importantes avanços nos estudos sobre formação e gestão de redes de cooperação e inovação entre pequenas e médias empresas e redes sociais. Se isso é particularmente verdadeiro, quando se trata de investigações voltadas para aumento de competitividade no mercado, o mesmo não se pode afirmar quando o objeto de estudo está centrado na ação de órgãos públicos que atuam na área social e, em particular, no setor saúde. 
A atuação colaborativa das diversas instituições ainda é incipiente. Por outro lado, esse quadro comporta um crescimento de demandas sociais que, de forma cada vez mais intensa, pressionam o Estado para busca de soluções 7 .

As estruturas tradicionais de organização, observadas a partir de 1900, podem ser caracterizadas por três principais modelos. O primeiro, chamado de estrutura funcional e identificado a partir do início do século XX, operava sua lógica baseado na especialização, com coordenação central. No período de 1920 a 1950, surgia a chamada estrutura divisional. São as divisões de produtos funcionando praticamente como empresas autônomas, porém ainda com controle central. Mais adiante, nas décadas de 60 e 70, em função da existência de mercados tanto estáveis como variáveis, aparecia a chamada estrutura matricial, que buscava uma combinação das formas anteriores. Com a crise econômica que se instala no início da década de 70, combinada com o esgotamento do sistema de produção em série, emergiram novas formas organizacionais estruturadas na idéia de rede 2 .

Levando-se em conta essa nova realidade, a concepção "Weberiana” de modelo organizacional, de integração vertical, não atende às necessidades requeridas para as novas formas de gestão, seja de pessoas, serviços ou produção ${ }^{8}$. Em contraposição, os modelos de redes sociais buscam a horizontalização e a articulação de demandas utilizando as modernas ferramentas da informação e da comunicação 9 .

São várias as abordagens empregadas que, dependendo do fundamento teórico utilizado, podem conduzir a um referencial conceitual diferente para a definição de rede. A pesquisa bibliográfica realizada mostrou que não há, entre os autores pesquisados, conceito universalmente aceito sobre o tema. Em sua discussão sobre empresa em rede, Castells 5 chama atenção a respeito da necessidade preliminar de se destacar dois tipos de organizações: as burocracias cuja reprodução de seu sistema de meios passa a ser seu objetivo principal e as empresas, nas quais os objetivos e suas mudanças modelam e (re)modelam de forma infinita a estrutura dos meios. Assinaladas essas diferenças, o autor propõe o que se chama de uma definição, não nominalista, de rede aquela forma específica de empresa cujo sistema de meios é constituído pela intersecção de segmentos de sistemas autônomos de objetivos. Isso quer dizer que os componentes da rede podem ser autônomos, mas também dependentes em relação à rede, podendo, portanto, ser parte de outras redes, inclusive de outros sistemas de meios que articulem outros objetivos. Abramovay 10 aponta que o termo rede é utilizado há muito tempo no campo da teoria das organizações como instrumento analítico capaz de auxiliar na compreensão de certas formas de organização coletiva que ultrapassam o campo das ciências sociais para incorporar novos elementos como, por exemplo, a ecologia da população.

Numa concepção ampliada, o conceito de rede pode ser aplicado a qualquer organização, na medida em que se entenda rede como o conjunto de relações que se estabelece com outros atores sociais. Dessa forma, é necessário desenvolver novos conceitos para gerenciamento das redes organizacionais 2 .

Redes também podem ser entendidas levando-se em conta a delimitação geográfica em que estão inseridas. Por exemplo, considerando-se algumas redes de cooperação científica e tecnológica, estabelecidas na América Latina, é possível vislumbrar, do ponto de vista geográfico, pelo menos cinco configurações. Primeiro seriam as redes de cooperação inter-regional entre os países da América Latina. Funcionariam através de atividades bilaterais, ou subregionais estabelecidas por relações interinstitucionais, interempresariais ou interpessoais. Outra configuração de rede seria a da cooperação hemisférica, que ocorre entre os países da América Latina e os Estados Unidos, Canadá e Caribe. Uma terceira configuração seria as redes de cooperação entre América Latina e Europa, tanto em nível bilateral como em bloco com a União Européia. Pode ainda ocorrer um quarto tipo de rede de cooperação, a iberoamericana, que se diferencia da anterior pela natureza de sua articulação, que se dá entre os países da América Latina com Espanha e Portugal. Por fim, teríamos as redes de cooperação com os países asiáticos do pacífico.

Outro esforço conceitual para a definição de redes é o apresentado por Abreu \& Goedert 8. Segundo eles, as organizações em rede na sua forma mais pura são grupos de pequenas e médias empresas que juntos fornecem um produto ou prestam um serviço. Os participantes, em função de suas especialidades, contribuem individualmente com ações que agregam valor ao produto oferecido. Isso significa, de acordo com Tarapanoff 11, imprimir aos produtos ou serviços produzidos uma diferenciação que os torna mais atraentes aos olhos do consumidor, quer seja em termos de qualidade, rapidez, durabilidade, assistência ou preço.

Ainda, conforme Abreu \& Goedert 8, de modo geral, uma rede de pequenas e médias empresas constitui-se de um conjunto de empre- 
sas, participando de um mesmo negócio, de forma autônoma e harmônica, operando em um regime de intensa cooperação, em que cada uma das firmas executa uma ou mais etapas do processo de produção, comercialização e distribuição de produtos/serviços, assim como a complementaridade de práticas gerenciais.

Casarotto \& Pires 12 ressaltam que, com as novas formas de atuação das empresas no atual ambiente de negócios envolvendo a terceirização, a parceria, a subcontratação e outras, surgiram novas estruturas de redes de empresas assim definidas: as redes topdown, que se caracterizam quando as empresas de menor porte fornecem direta e indiretamente sua produção a uma empresa mãe, através da terceirização, da parceria, da subcontratação e de outras formas de repasse da produção.

De outro lado, estariam as chamadas redes flexíveis de pequenas e médias empresas, que se caracterizam pela formação de um consórcio com objetivos comuns, em que cada empresa é responsável por parte do processo de produção, ou seja, o funcionamento em conjunto as tornaria uma grande empresa.

De acordo com Porter 13, redes também podem ser denominadas como aglomerados, ou seja, um agrupamento de empresas, geograficamente concentrado, inter-relacionadas e instituições correlatas numa determinada área, vinculadas por elementos comuns e complementares. Outra questão que se coloca nessa discussão conceitual são as aplicações do conceito de redes ou de organizações em rede. Em recente estudo, Saravia 14 nos indica algumas delas: como método de mapeamento de cadeias produtivas; como ferramenta destinada a verificar o funcionamento de setores regulados; além disso, sua aplicabilidade no campo da biologia. A partir dessa visão multifacetada, o autor conclui que, na prática, a rede é um conceito largamente operacional que permite construir novas realidades e modificar sistemas já existentes.

A articulação de diversos atores sociais, para gerar conhecimento e intervir numa realidade social, também pode ser entendida como rede. Essa idéia parte do princípio de que a realidade social precisa ser compreendida como sistema, pois os problemas são interligados e interdependentes. O modelo mecanicista de pensar não permite apreender as reais transformações sociais ora em curso. Para Junqueira 15, é uma nova visão de mundo que valoriza o pensamento intuitivo e não-linear centrado na cooperação e parceria. Os fundamentos dessa concepção estão na mudança do paradigma mecanicista para o ecológico e baseiam-se numa vi- são holística de mundo integrado e não como uma coleção de peças isoladas 16. Essas reflexões têm animado discussões a respeito de como o chamado pensamento complexo pode fazer emergir novas formas de compreender as transformações nos sistemas organizacionais e nos modelos de redes.

Transpondo esses fundamentos para o setor saúde do Brasil, podemos enxergar o SUS como uma rede, na qual acham-se inter-relacionadas organizações, pessoas e interesses. Conforme Junqueira 15, os indivíduos são inseridos em redes sociais, nas quais deixam de ser objetos para serem atores, responsáveis pela construção de um SUS, que se reproduz para garantir direitos de cidadania.

Para Jacobi ${ }^{9}$, as identidades de resistência são constituídas por atores que precisam construir formas que originalmente se manifestam em desacordo com a chamada nova ordem mundial. As ações são fundadas na busca de justiça social, no exercício da cidadania e numa transnacionalização das iniciativas civis.

O termo rede também pode ser utilizado para descrever processo de formação de políticas públicas que envolvam múltiplos nós (organizações) dotados de conexões conforme descrito por Misoczky 17.

Assim, o que se observa é que, independente do conceito de rede que se adote, verificamse algumas tendências como, por exemplo, a consolidação da cultura de cooperação. Notase também a busca da universalização da cooperação científica e tecnológica. Um número cada vez maior de atores é incorporado aos processos de inovação, sejam públicos ou privados e nos esquemas de cooperação. Essas tendências têm como conseqüência aumentar o papel estratégico da cooperação e seus impactos entre os elos de integração das redes.

A concepção de rede adotada para fins deste artigo baseia-se na idéia da chamada ecologia profunda desenvolvida por Capra 18. Partese do principio de que o mundo é um todo integrado, e não uma coleção de partes dissociadas. Reconhece-se também a independência fundamental de todos fenômenos e o fato de que, enquanto indivíduos e sociedades, estamos todos encaixados nos processos cíclicos da natureza, sendo, em última análise, dependentes desse processo. Vale dizer um entendimento de rede que inclui, para além da compreensão de um todo funcional e as interdependências entre suas partes, sua inserção no ambiente social. 


\section{Método}

Neste estudo, considera-se a REDEBLH como um conjunto de instituições que atuam, direta ou indiretamente, nas ações de promoção do aleitamento materno, coleta, processamento, controle de qualidade e distribuição de leite humano ordenhado pasteurizado. Funciona de forma autônoma e sem hierarquia organizacional formalmente estabelecida. Fundamentos como compromisso social, cooperação técnica, geração e apropriação do conhecimento são elementos inerentes à realização das ações desenvolvidas evidenciando a busca de processo contínuo de inovação tecnológica 1 .

Goedert 19, com base nos estudos de outros autores $3,12,21$, apresenta proposta de natureza exploratória para a identificação de oportunidades de aplicação do modelo de redes de inovação. Essa configuração de rede proporciona o conhecimento e a inovação tecnológica; a modernização gerencial; a difusão de novos serviços além da integração e cooperação de potencialidades. Ainda mais, uma rede de inovação também se relaciona com parceiros que possuem objetivos alinhados, seja do setor financeiro, governamental, científico-tecnológico e outros. Assumindo, em razão das características já apontadas na descrição do objeto de estudo, que a REDEBLH insere-se no sistema nacional de inovação em saúde, tal qual descrito em Gadelha 20, optou-se, neste estudo, pela adoção do caminho analítico proposto por Goe- dert 19. Isso implica, num primeiro momento, na identificação e categorização dos principais operadores dessa rede.

\section{Resultados e discussão}

Os atores envolvidos, os agentes externos, os monitores de avaliação (gatekeeping) e a explicitação de objetivos foram as categorias analíticas selecionadas. Na REDEBLH, pode-se, numa análise preliminar, identificar o perfil de seus integrantes diretos e indiretos (atores envolvidos), como apresentado na Tabela 2.

Os atores da Rede mantêm relações constantes entre si e também com atores de outras redes. Essa ação se concretiza através dos chamados nós de relacionamento. Adotando-se o desenho de rede sugerido por Goedert 19, podese vislumbrar, para a REDEBLH, uma configuração como a indicada na Figura 1. Nessa representação, ainda preliminar, e tentativa de identificação e contextualização dos diversos atores que nela interagem, observa-se a rede com olhar externo buscando entender a inter-relação e o papel dos diversos atores envolvidos.

A “externalização" (ameaças internas ou externas) pode ser entendida como, por exemplo, as possibilidades de mudanças na condução e formulação das políticas de saúde. De acordo com Goedert 19, o gatekeeping encarrega-se de apontar as informações de forma contínua na rede, além de realizar contatos com os diferen-

Tabela 2

Atores e descrição do perfil.

\begin{tabular}{|c|c|}
\hline Atores & Descrição \\
\hline $\begin{array}{l}\text { Sede da Rede, BLH, } \\
\text { Comissões de Aleitamento }\end{array}$ & $\begin{array}{l}\text { Sede da Rede localizada na FIOCRUZ, BLH localizados em cinco diferentes regiões geográficas do Brasil. } \\
\text { As comissões reúnem representantes de Estados e Municípios }\end{array}$ \\
\hline Instituições financeiras & A principal fonte de financiamento é o SUS (Ministério da Saúde, Secretarias Estaduais e Municipais de Saúde) \\
\hline Grandes empresas & $\begin{array}{l}\text { Além da FIOCRUZ, outras empresas já participam da Rede para geração de novos produtos, alavancagem de } \\
\text { pesquisas e investimentos. Já existem experiências inovadoras de parcerias oficializadas através de convênios, } \\
\text { dentre essas se pode citar: Empresa de Correios e Telégrafos e DATASUS }\end{array}$ \\
\hline Associações de classe & $\begin{array}{l}\text { A Sociedade Brasileira de Pediatria cumpre papel importante na disseminação de ações preventivas na área } \\
\text { infantil, o que a torna fundamental parceira para consolidação e ampliação da rede }\end{array}$ \\
\hline $\begin{array}{l}\text { Grupos de Pesquisa } \\
\text { \& Desenvolvimento }\end{array}$ & FIOCRUZ, Universidades e Institutos de Pesquisa \& Desenvolvimento \\
\hline $\begin{array}{l}\text { Organizações } \\
\text { não-governamentais }\end{array}$ & Amigas do Peito, Associação Brasileira de Profissionais de Banco de Leite Humano \\
\hline
\end{tabular}

BLH = Banco de Leite Humano; FIOCRUZ = Fundação Oswaldo Cruz; DATASUS = Departamento de Informação e Informática do SUS. 


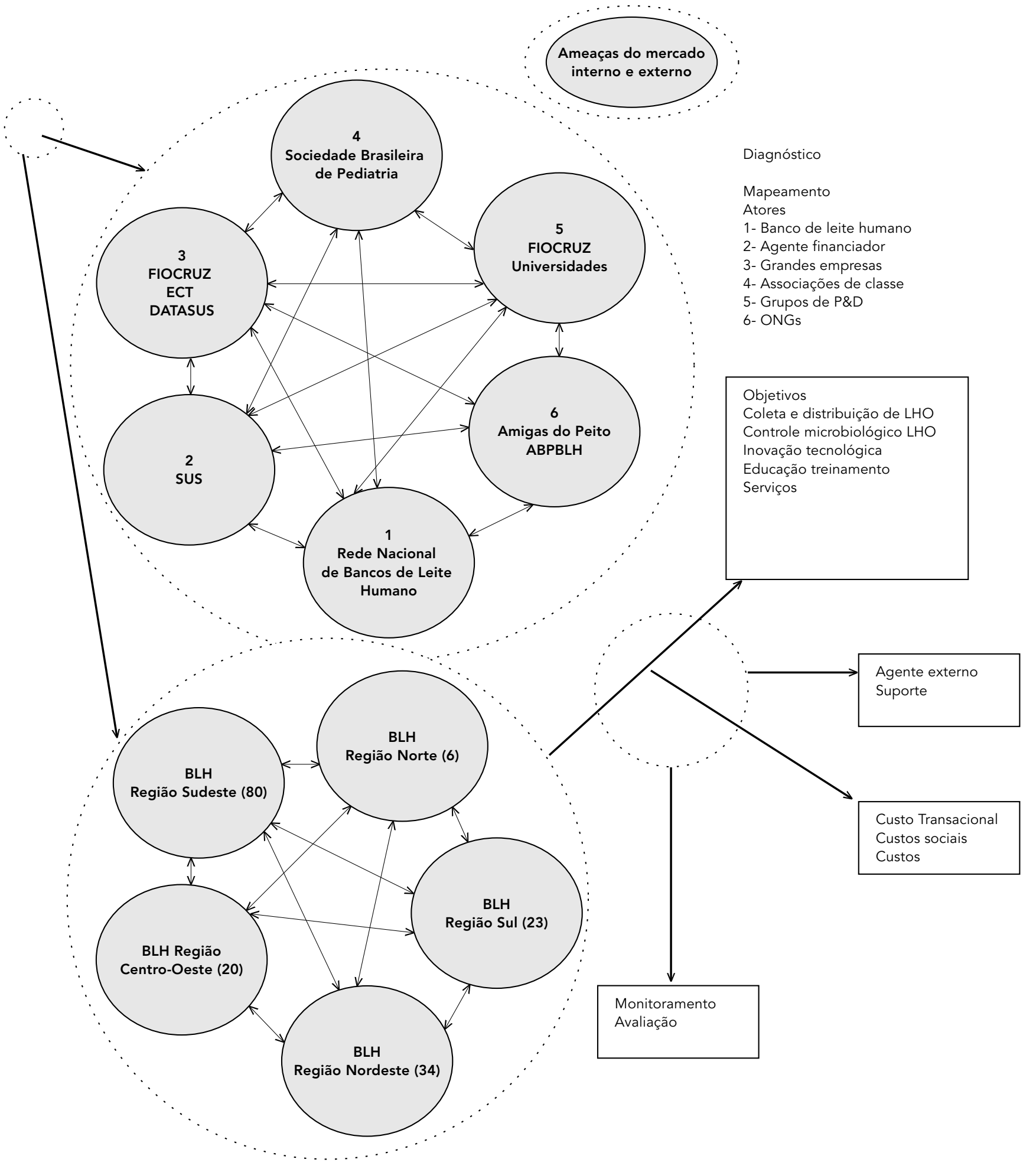

Adaptado da representação gráfica de rede proposta por Goedert 19

ABPBLH = Associação Brasileira de Profissionais de Banco de Leite Humano; FIOCRUZ = Fundação Oswaldo Cruz; ECT = Empresa

de Correios e Telégrafos; BLH = Banco de Leite Humano; ONGs = Organizações Não-governamentais; LHO = Leite Humano Ordenhado 
tes grupos técnicos dentro da rede e ligá-los aos demais centros tecnológicos, estabelecendo a cooperação entre eles. A geração e o compartilhamento do conhecimento também são atribuições de extrema importância para o desenvolvimento e o estabelecimento de oportunidades de melhoria.

No caso da REDEBLH, esses atores estão na equipe do Centro de Referência Nacional e nas Referências estaduais.

Identificou-se também que atividades de monitoramento e avaliação são ainda incipientes, necessitando de maior estruturação, do ponto de vista gerencial. Por outro lado, o controle de qualidade do leite humano distribuído é realizado de forma eficiente, pois são adotadas modernas técnicas de processamento e controle, sendo todo processo submetido ao monitoramento da vigilância sanitária local.

Com relação aos custos transacionais, sejam sociais ou econômicos, ainda são necessários estudos que possam apurá-los com maior detalhamento.

Os objetivos da REDEBLH estão explicitados (http:www.redeblh.fiocruz.br, acessado em 29/Abr/2004), porém acredita-se que, na prática, outros sejam estabelecidos pela própria dinâmica de seu funcionamento. Ou seja, a natureza deste formato operacional, intensivo na relação entre atores com base na solidariedade e cooperação, traz como resultado a incorporação permanente e, portanto, também dinâmica, de novos objetivos.

\section{Conclusões}

Por meio deste estudo, foi possível verificar que os resultados obtidos, de um modo geral, oferecem caminho analítico de utilidade para a discussão conceitual sobre o processo de gestão da REDEBLH. Avanços serão obtidos na medida em que outras investigações ampliem esse olhar para além do formalismo estático de identificação de atores. As doadoras voluntárias, equipes de saúde e o próprio SUS são atores que fazem a REDEBLH interagir no ambiente social. É preciso trazer para o escopo da análise esses elementos que lhe conferem animação.

Esse movimento analítico complementar deve implicar em contribuições para melhor compreender a gênese e a identidade funcional da REDEBLH. Vale dizer que, a partir do entendimento do processo de construção da REDEBLH e da identificação do papel dos atores e suas tramas, será possível avançar no desenho de novas estratégias de gestão.

A importância social da REDEBLH é incontestável. A incorporação de novos saberes que fundamentam a gestão é também, sem dúvida, uma prática a ser perseguida no caminho da modernização. Espera-se que o referencial teórico apresentado possa contribuir para a elaboração de novos estudos visando à consolidação e ampliação do posicionamento estratégico da Rede no campo da saúde pública nacional e no fortalecimento da cultura de cooperação.

\section{Resumo}

A Rede Nacional de Bancos de Leite Humano (REDEBLH), com sede na Fundação Oswaldo Cruz, experimenta rápido desenvolvimento. O trabalho desenvolvido foi reconhecido pela Organização Mundial de Saúde e distinguido, com o prêmio Sasakawa de Saúde - 2001, como o melhor projeto de saúde pública dentre os apresentados. Um dos grandes desafios atuais é dar continuidade ao desenvolvimento de competências que respondam aos desafios decorrentes de seu crescimento. Na realidade, busca-se uma nova estratégia de pensar as questões relacionadas a sua gestão. Este artigo tem como objetivos desenvolver um marco conceitual que possa contribuir para elaboração de um quadro teórico para novas estratégias de gestão da REDEBLH. Através da utilização de conceitos, buscase, à luz da tipologia de redes descrita na literatura especializada, identificar o perfil institucional da REDEBLH. A partir do entendimento de que é necessário identificar e compreender os processos que ocorrem no âmbito das redes para então pensar as questões relacionadas a sua gestão, foi utilizada como instrumento de análise proposta desenvolvida para formação de redes de inovação.

Bancos de Leite; Política de Saúde; Capacidade de Gestão 


\section{Colaboradores}

A concepção original do artigo bem como uma primeira versão foram elaboradas por P. R. S. Maia. Todos autores participaram das revisões e da elaboração do texto final.

\section{Referências}

1. Almeida JAG. Breastfeeding: a nature - culture hybrid. Rio de Janeiro: Fundação Oswaldo Cruz; 2001.

2. Peci A. Emergência e proliferação de redes organizacionais: marcando mudanças no mundo de negócios. Rev Adm Pública 1999; 33:7-24.

3. Rogers EM. Diffusion of innovation. New York: Free Press; 1995.

4. Freitas ME. Cultura organizacional: identidade, sedução e carisma? Rio de Janeiro: Editora FGV; 1999.

5. Castells M. A sociedade em rede. Rio de Janeiro: Paz e Terra; 2001

6. Latour B. Jamais fomos modernos. Rio de Janeiro. Editora 34; 2000.

7. Maia PRS. Construção de um modelo para medição e avaliação de programas institucionais de pesquisa e desenvolvimento tecnológico: produção do conhecimento científico na Fundação Oswaldo Cruz In: Anais do 21 Simpósio de Gestão da Inovação Tecnológica. [CD-ROM] São Paulo: Editora do Núcleo de Política e Gestão Tecnológica, Universidade de São Paulo; 2000.

8. Abreu AF, Goedert AR. Metodologia para formação de redes de inovação entre pequenas e médias empresas In: Anais do 21o Simpósio de Gestão da Inovação Tecnológica. [CD-ROM] São Paulo: Editora do Núcleo de Política e Gestão Tecnológica, Universidade de São Paulo; 2000.

9. Jacobi P. Meio ambiente e redes sociais: dimensões intersetoriais e complexidade na articulação de práticas coletivas. Rev Adm Pública 2000; 34: 131-58.

10. Abramovay R. A rede, os nós, as teias: tecnologias alternativas na agricultura. Rev Adm Pública 2000; 34:159-77.

11. Tarapanoff K, Araújo Jr. RH, Cormier PMJ. Sociedade de informação e inteligência em unidades de informação. Ciência da Informação 2000; 29: 91-100.

\section{Agradecimentos}

Para as professoras Suely Ferreira Deslandes e Virginia Alonso Hortale pelas importantes contribuições e permanente incentivo. A participação na oficina de autores promovida pela coordenação de pós-graduação em saúde da mulher e da criança do Instituto Fernandes Figueira, Fundação Oswaldo Cruz, foi fundamental para elaboração deste artigo.

12. Casarotto Filho N, Pires LH. Redes de pequenas e médias empresas e desenvolvimento local - estratégias para a conquista da competitividade global com base na experiência Italiana. São Paulo: Atlas; 1999.

13. Porter ME. Competição - on competition: estratégias competitivas essenciais. Rio de Janeiro: Campus; 1999.

14. Saravia H. Redes, organizações em rede e organizações virtuais. As novas configurações organizacionais. http://www.indeg.org/rbpg/index.html, acessado em 30/Jun/2003.

15. Junqueira LAP. Intersetorialidade, transetorialidade e redes sociais na saúde. Rev Adm Pública 2000; 34:35-45.

16. Silva AB, REBELO, LMB. A emergência do pensamento complexo nas organizações. Rev Adm Pública 2003; 37:777-96.

17. Misoczky MC. Redes e hierarquia: uma reflexão sobre arranjos de gestão na busca de equidade em saúde. Rev Adm Pública 2003; 37:335-54.

18. Capra F. A teia da vida. São Paulo: Editora Cultrix; 1996.

19. Goedert AR. Redes de inovação para pequenas e médias empresas: um estudo exploratório para o setor apícola catarinense [Dissertação de Mestrado]. Santa Catarina: Faculdade de Engenharia de Produção, Universidade Federal de Santa Catarina; 1999.

20. Gadelha CA, Quental C, Fialho BC. Sistema de inovação: uma abordagem sistêmica das indústrias da saúde. Cad Saúde Pública 2003; 19:47-59.

21. Tornatzky LG, Fleischer M. The process of technological innovation. Toronto: Lexington Books; 1990.

Recebido em 15/Jan/2004

Versão final reapresentada em 19/Mai/2004 Aprovado em 19/Jul/2004 